\title{
Solution of Hyperbolic Bioheat
}

F. S. Loureiro

felipe.loureiro@uff.edu.br Federal University of Juiz de Fora Department of Computer Science 36036-330 Juiz de Fora, MG, Brazi

\section{C. Wrobel}

Luiz.Wrobel@brunel.ac.uk Brunel University

School of Engineering and Design Uxbridge

UB8 3PH London, UK

\section{W. J. Mansur}

webe@coc.ufrj.br

Federal University of Rio de Janeiro Department of Civil Engineering 21945-970 Rio de Janeiro, RJ, Brazil
Transfer Problems by Numerical Green's Functions: The ExGA-Linear $\theta$ Method

This paper presents a time-domain formulation called Explicit Green's approach (ExGA) linear $\theta$ method for the solution of the bioheat equation. Starting from the hyperbolic bioheat equation, which includes the parabolic one as a special case, the linear method is incorporated into the standard ExGA time marching scheme. The numerical Green's function is firstly computed in the Laplace transform domain and then back-transformed to the time domain through the Stehfest inversion algorithm. The proposed formulation has the properties of stabilizing the results and suppressing numerical oscillations that appear in the presence of discontinuous solutions as assessed through the analysis of some bioheat transfer problems.

Keywords: numerical Green's function, bioheat, ExGA, time-marching scheme, Laplace transform

\section{Introduction}

Numerical methods have been widely used as a powerful tool for the solution of many problems in the thermo-biology field. There are several different mathematical models that can be used to describe the bioheat transfer process in living tissue (Rubinsky, 2006; Khanafer and Vafai, 2009). Among these, the Pennes' bioheat equation is frequently adopted in applications such as cancer hyperthermia therapies, cryosurgery, laser surgery, thermal diagnostics, skin burns and thermal comfort analysis. The literature reports a great deal of works on the numerical modelling of the parabolic Pennes equation considering different numerical techniques (Chan, 1992; Torvi and Dale, 1994; Liu and Xu, 2000; Dai, Yu and Nassar, 2004; Rubinsky, 2006; Presgrave, Guedes and Scofano Neto, 2009), e.g., finite difference, finite element and boundary element methods, each of them with its own advantages and disadvantages depending on the problem under consideration. However, there are some applications of extremely short time duration or at very low temperature (e.g., cryogenic surgery, laserinduced thermal damage, etc.) for which the parabolic Pennes bioheat equation, which assumes an infinite thermal speed of propagation according to Fourier's law, is not adequate and the mathematical model may be more accurately described by the hyperbolic bioheat equation (Lu, Liu and Zeng, 1998; Liu, Chen and Xu, 1999; Tunga, 2009; Özen, Helhel and Çerezci, 2008; Liu, 2008; $\mathrm{Xu}$, Seffen and Lu, 2008; Zhou, Zhang and Chen, 2009). The hyperbolic bioheat equation is characterised by the finite thermal speed of propagation of the thermal waves due to the application of a modified Fourier's law (Cattaneo, 1958).

Green's functions are an important tool in solving partial differential equations since the solution of a problem subjected to any kind of initial conditions, boundary conditions and internal heat generation can be obtained through integral equations once the Green's function is known. Both analytical and numerical techniques for the solution of heat conduction problems based upon Green's functions are well-documented (Beck, 1992; Wu and Chu, 1999; Wrobel, 2002). However, the solution of bioheat problems using Green's functions is not widely popular (Newman, Lele and

Paper received 30 January 2012. Paper accepted 14 May 2012 Technical Editor: Horácio Vielmo
Bowman, 1990; Chan, 1992; Gao B., Langer and Corry, 1995; Deng and Liu, 2002; Li, Liu and Yue, 2009), with few published papers which concentrate mainly on the parabolic Pennes equation. It is well known that one of the major drawbacks in the solution of partial differential equations by Green's function-based techniques is the lack of analytical Green's functions for complicated geometries and/or variable material properties. To circumvent these disadvantages, a formulation named Explicit Green's Approach (ExGA) was proposed in Mansur et al. (2007) and Loureiro (2007). The technique is based on the use of numerical Green's functions instead of analytical ones, and combine concepts from both finite element method (FEM) (Hughes, 1987) and time-domain boundary element method (BEM) (Mansur, 1983; Wrobel, 2002), as demonstrated by Loureiro, Mansur and Vasconcellos (2009) and Loureiro and Mansur (2010). Hence, the flexibility offered by the ExGA method makes it very general and applicable to many classes of problems.

The main aim of this paper is to present a novel time-domain formulation for the solution of the hyperbolic bioheat equation. The proposed time-domain formulation combines the ExGA methodology (Loureiro et al., 2010) with the linear $\theta$ method presented in $\mathrm{Yu}$ et al. (1998) in a BEM context, giving rise to a new formulation denominated ExGA-linear $\theta$ method. Following the works of Loureiro, Mansur and Vasconcellos (2009) and Loureiro and Mansur (2009a), a Laplace transform technique in conjunction with the FEM is applied to the Green's function equation; subsequently, the Gaver-Stehfest Laplace inversion algorithm (Stehfest, 1970) is employed to compute the Green's function in the time-domain. Since Green's functions are firstly formulated taking into account the Laplace transform and then incorporated into the ExGA-linear $\theta$ time-marching scheme, a hybrid time-Laplace technique is derived, resulting in the ExGA-Stehfest linear $\theta$ method. The ExGA-linear $\theta$ method is suited not only to stabilize the numerical results but also to suppress numerical (spurious) oscillations that may appear near discontinuous solutions in the hyperbolic model. Besides, its computer implementation is very straightforward. The applicability, potentialities and accuracy of the technique are demonstrated by considering some numerical examples with discontinuous solutions in the hyperbolic model. 


\section{Nomenclature}

C = specific heat of tissue, $J \cdot g^{-1} \cdot{ }^{0} C^{-1}$

$c_{b} \quad=$ specific heat of blood, $J \cdot g^{-1} \cdot{ }^{0} C^{-1}$

$\mathbf{G}(t) \quad=$ Green's matrix

$\overline{\mathbf{g}}_{j}(s)=$ Green's vector in the Laplace domain

$k=$ thermal conductivity of tissue, $W \cdot \mathrm{mm}^{-1} .{ }^{0} \mathrm{C}^{-1}$

$N(\mathbf{x}) \quad=$ interpolation function

$Q_{r} \quad=$ spatial heating, $W \cdot \mathrm{mm}^{-3}$

$Q_{m} \quad=$ metabolic heat of tissue, $W \cdot \mathrm{mm}^{-3}$

$\bar{q}(\mathbf{x}, t)=$ prescribed boundary heat flux,$W \cdot \mathrm{mm}^{-2}$

$T(\mathbf{x}, t)=$ tissue temperature, ${ }^{\circ} \mathrm{C}$

$T_{0}(\mathbf{x})=$ initial temperature, ${ }^{\circ} \mathrm{C}$

$\bar{T}(\mathbf{x}, t)=$ prescribed boundary temperature, ${ }^{\circ} \mathrm{C}$

$T_{b}(\mathbf{x}, t)=$ blood temperature, ${ }^{\circ} \mathrm{C}$

$\mathbf{T}(t) \quad=$ temperature vector

$\dot{\mathbf{T}}(t)=$ time derivative of the temperature vector

$\mathbf{T}_{p}(t)=$ particular solution vector

$V^{h} \quad=$ finite element space

$w_{b} \quad=$ perfusion rate of blood, $g \cdot \mathrm{mm}^{-3} \cdot \mathrm{s}^{-1}$

$W^{h} \quad=$ weighting function

\section{Greek Symbols}

$\begin{array}{ll}\delta_{k j} & =\text { Kronecker delta } \\ \delta(\cdot) & =\text { dirac delta function } \\ \Delta t & =\text { time step size, } S \\ \rho & =\text { density of tissue, } \text { g.mm }^{-3} \\ \theta & =\text { linear } \theta \text { method parameter } \\ \tau_{r} & =\text { thermal relaxation time, } s \\ \lambda_{i} & =\text { eigenvalue } \\ \Gamma & =\text { Lipschitz boundary } \\ \Omega & =\text { open bounded domain }\end{array}$

\section{Mathematical Equations}

Let $\Omega \subset \mathbb{R}^{d}$ be an open bounded domain with Lipschitz boundary $\Gamma=\partial \Omega$, where $d$ is the number of spatial dimensions, and let $I=\left(0, t_{f}\right]$ be the total time interval of the analysis, with $t_{f}>0$. The governing equation for hyperbolic bioheat transfer problems reads (Liu, Chen and $\mathrm{Xu}, 1999$ ):

$$
\begin{aligned}
& \nabla \cdot(k \nabla T(\mathbf{x}, t))+w_{b} c_{b}\left(T_{b}(\mathbf{x}, t)-T(\mathbf{x}, t)\right)+b(\mathbf{x}, t) \\
& =\tau_{r} \rho c \ddot{T}(\mathbf{x}, t)+\left(\rho c+\tau_{r} w_{b} c_{b}\right) \dot{T}(\mathbf{x}, t)
\end{aligned}
$$

where $b(\mathbf{x}, t)=Q_{m}(\mathbf{x}, t)+Q_{r}(\mathbf{x}, t)+\tau_{r}\left(\dot{Q}_{m}(\mathbf{x}, t)+\dot{Q}_{r}(\mathbf{x}, t)\right)$.

In Eq. (1), $\nabla$ denotes the gradient operator, $T_{b}(\mathbf{x}, t)$ is the blood temperature and $T(\mathbf{x}, t)$ is the tissue temperature, with over dots indicating derivatives with respect to time. Moreover, $k, \rho$ and $c$ stand for thermal conductivity, density and specific heat of tissue; $c_{b}$ and $w_{b}$ are the specific heat and perfusion rate of blood, respectively. The volumetric heat $b(\mathbf{x}, t)$ contains the metabolic and spatial heating terms $Q_{m}(\mathbf{x}, t)$ and $Q_{r}(\mathbf{x}, t)$, as well as their time derivatives; $\tau_{r}$ is the thermal relaxation time of the biological system. The boundary $\Gamma$ consists of a part $\Gamma_{1}$ with prescribed temperature and a part $\Gamma_{2}$ with prescribed heat flux, with its unit outward normal vector represented by $\mathbf{n}$, such that $\Gamma=\Gamma_{1} \cup \Gamma_{2}$ and $\Gamma_{1} \cap \Gamma_{2}=\varnothing$, i.e.:

$$
\begin{aligned}
& T(\mathbf{x}, t)=\bar{T}(\mathbf{x}, t) \text { on } \Gamma_{1} \times I \\
& k \nabla T(\mathbf{x}, t) \cdot \mathbf{n}=\bar{q}(\mathbf{x}, t) \text { on } \Gamma_{2} \times I
\end{aligned}
$$

Initial conditions are given by

$$
\begin{aligned}
& T(\mathbf{x}, 0)=T_{0}(\mathbf{x}) \text { in } \Omega \\
& \dot{T}(\mathbf{x}, 0)=\dot{T}_{0}(\mathbf{x}) \text { in } \Omega
\end{aligned}
$$

The heat flux obeys the modified Fourier's law given by $\mathbf{q}(\mathbf{x}, t)+\tau_{r} \partial \mathbf{q}(\mathbf{x}, t) / \partial t=-k \nabla T(\mathbf{x}, t) \quad$ (Cattaneo, 1958), which differs from the classical Fourier conduction law by an additional term including the time rate of change of the heat flux multiplied by $\tau_{r}$. Notice that the well-known Pennes bioheat equation, which is of parabolic type, is readily obtained by assuming $\tau_{r}=0$ in Eq. (1).

Due to the lack of analytical Green's functions for general bioheat problems (i.e., with non-homogeneous media, complexshaped geometries, etc.), the solution of Eqs. (1)-(5) for the tissue temperature is derived by employing the Explicit Green's Approach (ExGA) formulation. In this way, the expression for the tissue temperature by means of the ExGA formulation is given by (more details on the main steps required for the derivation of the ExGA expression can be found in Loureiro et al., 2010; Loureiro and Mansur, 2010):

$$
\begin{gathered}
\tilde{\mathbf{T}}(t)=\left(\mathbf{G}\left(t-t_{0}\right)\left(\mathbf{M}+\tau_{r} \mathbf{C}\right)+\tau_{r} \dot{\mathbf{G}}\left(t-t_{0}\right) \mathbf{M}\right) \tilde{\mathbf{T}}\left(t_{0}\right)+ \\
\tau_{r} \mathbf{G}\left(t-t_{0}\right) \mathbf{M} \dot{\tilde{\mathbf{T}}}\left(t_{0}\right)+\int_{t_{0}}^{t} \mathbf{G}(t-\tau) \mathbf{F}(\tau) d \tau
\end{gathered}
$$

where $\mathbf{G}\left(t-t_{0}\right)$ and $\dot{\mathbf{G}}\left(t-t_{0}\right)$ represent the Green's function and its time derivative written in matrix form for the discrete system as will be discussed further. The matrix and vector entries appearing in Eq. (6) are defined as:

$$
\begin{aligned}
& M_{j l}=\int_{\Omega} N_{j}(\mathbf{y}) \rho c N_{l}(\mathbf{y}) d \Omega_{\mathbf{y}} \\
& C_{j l}=\int_{\Omega} N_{j}(\mathbf{y}) w_{b} c_{b} N_{l}(\mathbf{y}) d \Omega_{\mathbf{y}}
\end{aligned}
$$




$$
\begin{gathered}
F_{j}(\tau)=\int_{\Omega} N_{j}(\mathbf{y})\left(b(\mathbf{y}, \tau)+w_{b} c_{b} T_{b}(\mathbf{y}, \tau)\right) d \Omega_{\mathbf{y}}+ \\
\int_{\Gamma_{2}} N_{j}(\mathbf{y}) \bar{q}(\mathbf{y}, \tau) d \Gamma_{\mathbf{y}}-\bar{F}_{j}(\tau)
\end{gathered}
$$

with $\bar{F}_{j}(\tau)$ being the vector containing the contribution of the prescribed temperature on the boundary $\Gamma_{1}$. The vector components are defined as:

$$
\begin{aligned}
\bar{F}_{j}(\tau)= & \int_{\Omega} \nabla N_{j}(\mathbf{y}) \cdot k \nabla N_{l}(\mathbf{y}) d \Omega_{\mathbf{y}} \bar{T}_{l}(\tau)+ \\
& \int_{\Omega} N_{j}(\mathbf{y}) w_{b} c_{b} N_{l}(\mathbf{y}) d \Omega_{\mathbf{y}} \bar{T}_{l}(\tau)+ \\
& \int_{\Omega} N_{j}(\mathbf{y})\left(\rho c+\tau_{r} w_{b} c_{b}\right) N_{l}(\mathbf{y}) d \Omega_{\mathbf{y}} \frac{\partial \bar{T}_{l}(\tau)}{\partial \tau}+ \\
& \tau_{r} \int_{\Omega} N_{j}(\mathbf{y}) \rho c N_{l}(\mathbf{y}) d \Omega_{\mathbf{y}} \frac{\partial^{2} \bar{T}_{l}(\tau)}{\partial \tau^{2}}
\end{aligned}
$$

It is worth mentioning that if the convective boundary condition is imposed, little modifications must be accomplished in the proposed formulation. Taking into account the modified Fourier's Law, the convective boundary condition can be expressed as $k \nabla T(\mathbf{x}, t) \cdot \mathbf{n}=-h\left(\tau_{r} \partial T(\mathbf{x}, t) / \partial t+T(\mathbf{x}, t)-T_{a}(\mathbf{x}, t)\right), \quad$ where $\quad h$ denotes the convective heat transfer coefficient and $T_{a}$ the temperature of the medium surrounding the convective boundary. In this way, the known term is implemented replacing $\bar{q}(\mathbf{y}, \tau)$ by $h T_{a}(\mathbf{y}, \tau)$ into Eq. (9) while the boundary integral $\int_{\Gamma_{c}} N_{j}(\mathbf{y}) h N_{l}(\mathbf{y}) \Gamma_{\mathbf{y}}$ is appended to the matrix $\mathbf{C}$ (Eq. (8)). In addition, a homogeneous convective boundary condition must be adopted to compute the Green's function.

Although the convolution integral offers a more general approach, it is possible to replace it by a particular solution related to the external heat load (Loureiro and Mansur, 2009a-b). Thus, Eq. (6) can be rewritten in terms of the particular solution as follows:

$$
\begin{aligned}
\tilde{\mathbf{T}}(t)= & \left(\mathbf{G}\left(t-t_{0}\right)\left(\mathbf{M}+\tau_{r} \mathbf{C}\right)+\tau_{r} \dot{\mathbf{G}}\left(t-t_{0}\right) \mathbf{M}\right)\left(\tilde{\mathbf{T}}\left(t_{0}\right)-\tilde{\mathbf{T}}_{p}\left(t_{0}\right)\right) \\
& +\tau_{r} \mathbf{G}\left(t-t_{0}\right) \mathbf{M}\left(\dot{\tilde{\mathbf{T}}}\left(t_{0}\right)-\dot{\tilde{\mathbf{T}}}_{p}\left(t_{0}\right)\right)+\tilde{\mathbf{T}}_{p}(t)
\end{aligned}
$$

where $\tilde{\mathbf{T}}_{p}(t)$ is the particular solution vector corresponding to the external heat load vector $\mathbf{F}(t)$ (see, for instance, Loureiro and Mansur (2009a,b) for additional details on how to obtain the particular solution for different types of time shape function).

The major difference between the use of the particular solution and the time-domain convolution integral lies in the way in which each technique accomplishes the contribution of the heat load vector into the final solution. In the particular solution technique, the contribution is taken into account analytically as long as the heat load shape function is correctly represented, whereas a quadrature rule must be used in the convolution integral since the Green's matrix is computed numerically, generating a certain degree of error in the final solution.

\section{The ExGA-Linear $\theta$ Time-Marching Scheme}

In the original linear $\theta$ method proposed by Yu et al. (1998), a linear time variation for both the potential and the flux was adopted into the standard time-domain boundary element method (TDBEM), aiming at producing more stable results in the solution of the scalar wave equation. Here, the linear $\theta$ method is applied to the ExGA formulation, but, unlike the work of Yu et al. (1998), a linear time variation for the temperature and its time derivative is proposed. Consider a partition of the total time interval $\left[0, t_{f}\right]$ into $N$ equal time intervals, i.e., $\left[0, t_{f}\right]=\bigcup_{k=0}^{N-1}\left[t_{k}, t_{k+1}\right]$ with $0=t_{0}<t_{1}<\ldots<t_{N}=t_{f}, \quad \Delta t=t_{k+1}-t_{k}=t_{f} / N, \quad t_{k}=k \Delta t \quad$ and $t_{k+1}=(k+1) \Delta t$. The main idea of the ExGA-linear $\theta$ method is to evaluate firstly the solution at time $t_{k+\theta}=(k+\theta) \Delta t$ from the previous known solution at time $t_{k}$ in a recursive manner. Hence, in order to establish recursive expressions, it is first necessary to obtain the time derivative expression of the temperature vector by differentiating Eq. (11). Then, writing both the temperature and its time derivative vectors for the time interval $\left[t_{k}, t_{k+\theta}\right]$, i.e., replacing $t_{0}$ by $t_{k}$ and $t$ by $t_{k+\theta}$, with the solution at time $t_{k}$ playing the role of an initial condition for the calculation of the solution at time $t_{k+\theta}$, one obtains:

$$
\begin{aligned}
\tilde{\mathbf{T}}^{k+\theta}= & \left(\mathbf{G}(\theta \Delta t)\left(\mathbf{M}+\tau_{r} \mathbf{C}\right)+\tau_{r} \dot{\mathbf{G}}(\theta \Delta t) \mathbf{M}\right)\left(\tilde{\mathbf{T}}^{k}-\tilde{\mathbf{T}}_{p}^{k}\right)+ \\
& \tau_{r} \mathbf{G}(\theta \Delta t) \mathbf{M}\left(\dot{\tilde{\mathbf{T}}}^{k}-\dot{\tilde{\mathbf{T}}}_{p}^{k}\right)+\tilde{\mathbf{T}}_{p}^{k+\theta} \\
\dot{\tilde{\mathbf{T}}}^{k+\theta}= & -\mathbf{G}(\theta \Delta t)(\mathbf{K}+\mathbf{C})\left(\tilde{\mathbf{T}}^{k}-\tilde{\mathbf{T}}_{p}^{k}\right)+\tau_{r} \dot{\mathbf{G}}(\theta \Delta t) \mathbf{M}\left(\dot{\tilde{\mathbf{T}}}^{k}-\dot{\tilde{\mathbf{T}}}_{p}^{k}\right)+ \\
& \dot{\tilde{\mathbf{T}}}_{p}^{k+\theta}
\end{aligned}
$$

In order to simplify the notation, the superscripts $k+\theta$ and $k$ in the above expressions represent values at the times $t_{k+\theta}$ and $t_{k}$, i.e., $\quad \tilde{\mathbf{T}}^{k+\theta} \equiv \tilde{\mathbf{T}}\left(t_{k+\theta}\right)$ and $\tilde{\mathbf{T}}^{k} \equiv \tilde{\mathbf{T}}\left(t_{k}\right)$, respectively. Finally, the desired solution at time step $t_{k+1}$ is then calculated using a linear interpolation within the time interval $\left[t_{k}, t_{k+\theta}\right]$, that is

$$
\begin{aligned}
\tilde{\mathbf{T}}^{k+1} & =\frac{1}{\theta} \tilde{\mathbf{T}}^{k+\theta}+\frac{\theta-1}{\theta} \tilde{\mathbf{T}}^{k} \\
\dot{\tilde{\mathbf{T}}}^{k+1} & =\frac{1}{\theta} \dot{\tilde{\mathbf{T}}}^{k+\theta}+\frac{\theta-1}{\theta} \dot{\tilde{\mathbf{T}}}^{k}
\end{aligned}
$$

where $\theta \geq 1$ is a free parameter employed either to control numerical oscillations or to stabilize the numerical solution, as will be discussed in the time-domain Green's function and selection of the parameter $\theta$ sub-section, as well as in the numerical aspects and applications section.

Because a recursive time-marching scheme is adopted as indicated by Eq. (12), the Green's function and its time derivative matrices need to be computed only once at time $t_{\theta}=t_{k+\theta}-t_{k}=\theta \Delta t$; additionally, the particular solution must be specified only within the 
time interval $\left[t_{k}, t_{k+\theta}\right]$. Notice that, to avoid the computation of the second time derivative of the Green's matrix, the relation $\dot{\mathbf{G}}(\theta \Delta t)\left(\mathbf{M}+\tau_{r} \mathbf{C}\right)+\tau_{r} \ddot{\mathbf{G}}(\theta \Delta t) \mathbf{M}=-\mathbf{G}(\theta \Delta t)(\mathbf{K}+\mathbf{C})$ originated by considering equilibrium equation concerning the Green's function at time $t_{\theta}=\theta \Delta t$ is adopted, where entries of the matrix $\mathbf{K}$ are given by

$$
K_{j l}=\int_{\Omega} \nabla N_{j}(\mathbf{y}) \cdot k \nabla N_{l}(\mathbf{y}) d \Omega_{\mathbf{y}}
$$

Equation (12) requires the computation of both the temperature and its time derivative vectors to carry out the time-marching procedure. For the parabolic bioheat equation, however, the timemarching process is evaluated only with the temperature vector as $\tau_{r}=0$ in Eq. (12), yielding:

$$
\tilde{\mathbf{T}}^{k+1}=\mathbf{G}(\Delta t) \mathbf{M}\left(\tilde{\mathbf{T}}^{k}-\tilde{\mathbf{T}}_{p}^{k}\right)+\tilde{\mathbf{T}}_{p}^{k+1}
$$

As the Pennes' equation produces smooth solutions, there is no mathematical justification to adopt the linear $\theta$ method for its solution, and $\theta=1$ is used in all calculations.

\section{Laplace Transform FEM Formulation to Compute the Green's Function Matrices}

This section describes the procedures for computing the numerical Green's function. First, the Green's function equation in the Laplace domain and its discretisation by means of the standard Galerkin finite element technique are briefly discussed. In the sequence, the set of coupled algebraic equations in the transformed domain is solved back to the time-domain by numerical Laplace inversion algorithms and the selection of the parameter $\theta$ is analyzed.

\section{Green's function in the Laplace transform domain}

Let $V^{h}$ be the finite element space of commonly continuous piecewise polynomials on $\Omega$. The classical Galerkin finite element method (Hughes, 1987) when applied to the Green function equation with homogeneous boundary conditions in the Laplace transform domain can be stated as:

$$
\begin{aligned}
& \text { find } \bar{G}^{h} \in V^{h} \text { such that } \\
& \int_{\Omega} W^{h}(\mathbf{x})\left(\tau_{r} \rho c s^{2}+\left(\rho c+\tau_{r} w_{b} c_{b}\right) s+w_{b} c_{b}\right) \bar{G}^{h}\left(\mathbf{x}, \mathbf{y}_{j}, s\right) d \Omega_{\mathbf{x}}+ \\
& \int_{\Omega} \nabla W^{h}(\mathbf{x}) \cdot k \nabla \bar{G}^{h}\left(\mathbf{x}, \mathbf{y}_{j}, s\right) d \Omega_{\mathbf{x}}=\int_{\Omega} W^{h}(\mathbf{x}) \delta\left(\mathbf{x}-\mathbf{y}_{j}\right) d \Omega_{\mathbf{x}} \\
& \forall W^{h} \in V^{h}
\end{aligned}
$$

where $W^{h}(\mathbf{x})=\sum N_{i}(\mathbf{x}) W_{i}$ stands for the weighting function and $\bar{G}^{h}\left(\mathbf{x}, \mathbf{y}_{j}, s\right)=\sum_{k} N_{k}(\mathbf{x}) \bar{G}_{k j}(s) \quad$ is the Green's function approximation in the transformed domain for a concentrated heat source applied at the nodal point $\mathbf{y}_{j}$ of the finite element mesh. Introducing the vector of the Green's function nodal values in the transformed domain $\overline{\mathbf{g}}_{j}(s)=\left(\bar{G}_{1 j}, \ldots, \bar{G}_{k j}, \ldots\right)^{T}$, Eq. (15) can be rewritten in an equivalent matrix form as:

$$
\left(\tau_{r} \mathbf{M} s^{2}+\left(\mathbf{M}+\tau_{r} \mathbf{C}\right) s+\mathbf{C}+\mathbf{K}\right) \overline{\mathbf{g}}_{j}(s)=\mathbf{1}_{j}
$$

where the matrices are the same as those defined previously and $\mathbf{1}_{j}$ with entries $\delta_{k j}$ denotes a unit base vector. Finally, the full Green's matrix in the Laplace domain is constructed from Green's vectors $\overline{\mathbf{g}}_{j}(s)$ as $\overline{\mathbf{G}}(s)=\left(\overline{\mathbf{g}}_{1}(s), \overline{\mathbf{g}}_{2}(s), \ldots, \overline{\mathbf{g}}_{j}(s), \ldots\right)$.

\section{Time-domain Green's function and selection of the parameter $\theta$}

The nodal values of the time-dependent Green's function can be obtained from their values in the transformed domain, Eq. (16), by means of an inverse Laplace transform. In the present paper, the Gaver-Stehfest is employed to find a solution in the time-domain. Thus, the inversion of the Green's vector at time $t=\theta \Delta t$, adopting the well-known Gaver-Stehfest algorithm (from now on referred only as Stehfest), can be written as (Stehfest, 1970) (recall that Green's functions only need to be computed at time $t=\theta \Delta t$ ):

$$
\mathbf{g}_{j}(\theta \Delta t)=\frac{\ln 2}{\theta \Delta t} \sum_{i=1}^{N} V_{i} \overline{\mathbf{g}}_{j}\left(\frac{\ln 2}{\theta \Delta t} i\right)
$$

where

$$
V_{i}=(-1)^{N / 2+i} \sum_{k=\lfloor(i+1) / 2\rfloor}^{\min (i, N / 2)} \frac{k^{N / 2}(2 k) !}{(N / 2-k) ! k !(k-1) !(i-k) !(2 k-i) !}
$$

with $N$ being an even number.

The reason for the use of the Stehfest algorithm is based on the following advantages, namely: i) easy computational implementation; ii) just one free parameter, i.e., the number of summation terms $N$ is required; and iii) only real arithmetic operations are carried out; thus, making the Stehfest algorithm more computationally effective than other inversion algorithms based on complex arithmetic operations which generally have more than one free parameter to be selected. Differently from the standard Laplace formulation, in the present hybrid method, the external thermal vector $\mathbf{F}(t)$ is taken into account directly in the time domain, avoiding the requirement of the application of the Laplace transform, which, in general, is also done numerically.

The remaining issue is concerned with the selection of the correct values for the parameter $\theta$ and its relation with the number of summation terms $N$. Let $\overline{\mathbf{M}}=\tau_{r} \mathbf{M}, \quad \overline{\mathbf{K}}=\mathbf{C}+\mathbf{K} \quad$ and $\overline{\mathbf{C}}=\mathbf{M}+\tau_{r} \mathbf{C}$ and owing to the fact that the condition $\overline{\mathbf{C}} \overline{\mathbf{M}}^{-1} \overline{\mathbf{K}}=\overline{\mathbf{K}} \overline{\mathbf{M}}^{-1} \overline{\mathbf{C}}$ is satisfied, a diagonalization procedure can be applied to decouple Eqs. (12)-(13) and (16). Hence, the parameter $\theta$ values are determined taking into account the stability analysis of following amplification matrix obtained from Eqs. (12)-(13), writing the solution at time $t_{k+1}$ in terms of the solution at time $t_{k}$ :

$$
\mathbf{A}=\left(\begin{array}{cc}
\frac{1}{\theta}\left(\dot{g}(\theta \Delta t)+c_{i} g(\theta \Delta t)\right)+(\theta-1) / \theta & \frac{1}{\theta} g(\theta \Delta t) \\
-\frac{1}{\theta} \omega_{i}^{2} g(\theta \Delta t) & \frac{1}{\theta} \dot{g}(\theta \Delta t)+(\theta-1) / \theta
\end{array}\right)
$$


where $\omega_{i}^{2}=\lambda_{i}$ with $\lambda_{i}$ being an eigenvalue, solution of the generalized eigenvalue problem given by $\lambda \overline{\mathbf{M}} \boldsymbol{\varphi}=\overline{\mathbf{K}} \boldsymbol{\varphi}$.

To ensure an unconditionally stable method without the need of computing eigenvalues and eigenvectors, the condition for the spectral radius of matrix $\mathbf{A}$ given by $\rho(\mathbf{A}) \leq 1$ must hold for all the range $\omega_{i} \Delta t$ and $c_{i}>0$. This upper bound stability requirement is achieved by selecting $\theta \geq 1.1$ for $N=8$ and $\theta>1.05$ for $10 \leq N \leq 16$. Once the stability requirement is hold, a time step must be selected in order to carry out the time marching procedure. The time step $\Delta t$ is readily selected bearing in mind the dimensionless parameter $\beta=v \Delta t / l_{e}$, where $v=\sqrt{k /(\rho c \tau)}$ stands for the thermal wave velocity and $l_{e}$ the characteristic element length, which is easily estimated by $l_{e}=\sqrt{\int_{\Omega_{e}} d \Omega}$. Although the proposed method is unconditionally stable for the $\theta$ values discussed above, the value of $\beta$ should not be large; otherwise, the overall accuracy of the proposed method may be deteriorated during the time-marching process.

\section{Numerical Aspects and Applications}

In this section, some numerical transient bioheat applications are presented, illustrating the potentialities of the proposed formulation. Initially, a uniform biological tissue model is analyzed and numerical results are compared to the available analytical solution in order to show the accuracy of the ExGA-linear $\theta$ method; subsequently, aiming to demonstrate the applicability of the ExGA method to deal with heterogeneous media, a triple layer skin model is discussed. Finally, the thermal behavior in a square biological body subjected to an initial temperature distribution is investigated. For all the numerical simulations that follow, the solution is written in terms of the elevated temperature $T_{s}(\mathbf{x}, t)=T(\mathbf{x}, t)-T_{b}$ with $T_{b}=37^{\circ} \mathrm{C}$. Furthermore, numerical results obtained by the proposed hybrid time-Laplace technique, namely, ExGA-Stehfest linear $\theta$ method ( $N=10$ is employed in all the examples except in Fig. 4 where a convergence analysis is performed) are compared to those of standard FEM formulations employing HHT- $\alpha$ and CrankNicolson time marching schemes (Hughes, 1987), as well as the standard Stehfest Laplace inversion algorithm.

\section{Homogeneous biological tissue model}

The first application is concerned with the analysis of a homogeneous biological tissue at null initial elevated temperature $T_{s 0}(\mathbf{x})=0.0^{\circ} \mathrm{C}$ subjected to a sudden temperature rise at the skin surface, with the rest of the boundary considered as adiabatic. A sketch of the model and the finite element mesh constructed with 4311 four-node quadrilateral elements are depicted in Fig. 1. It is assumed that, far from skin surface, the tissue is not affected by the prescribed temperature; therefore, a coarse mesh is constructed near the core body. The thermal properties of the tissue and blood are $\rho=1.0 \times 10^{-3} \mathrm{~g} . \mathrm{mm}^{-3}, c=$ $c_{b}=4.2 \mathrm{~J} . \mathrm{g}^{-1} .{ }^{\circ} \mathrm{C}^{-1}, k=5.0 \times 10^{-4} \mathrm{~W} . \mathrm{mm}^{-1} \cdot{ }^{\circ} \mathrm{C}^{-1}$ and $w_{b}=5.0 \times$ $10^{-7} \mathrm{~g} . \mathrm{mm}^{-3} \cdot \mathrm{s}^{-1}$. The time step is chosen such that $\beta=1$ with $l_{e}$ being the smallest characteristic element length, yielding $\Delta t=0.4 \mathrm{~s}$.

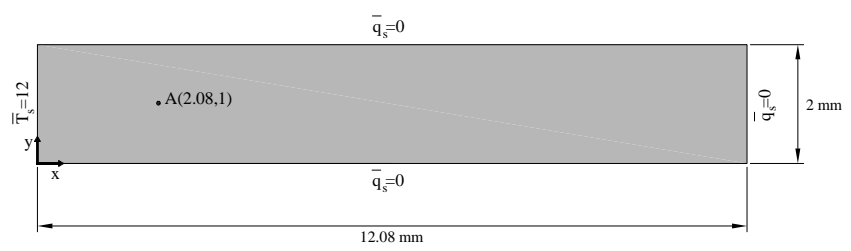

(a)

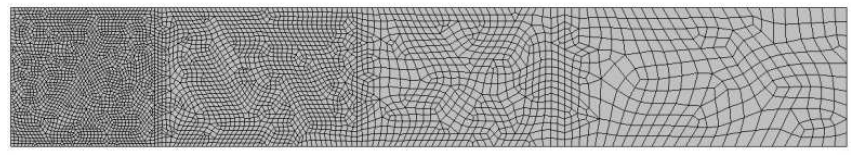

(b)

Figure 1. Homogeneous biological tissue body: (a) geometry and boundary conditions; (b) domain discretization.

In spite of its geometrical and load simplicity, this example is an important benchmark and quite difficult to solve numerically due to the thermal wave with discontinuous front caused by the suddenly applied temperature when $\tau_{r}>0$; besides, the analytical solution is available in the literature and its expression is given by (Liu, Chen and $\mathrm{Xu}, 1999$ ):

$$
\begin{aligned}
& T(x, t)= \frac{\bar{T}_{s} \cosh \left(\sqrt{\frac{w_{b} c_{b}}{k}}(x-L)\right)}{\cosh \left(\sqrt{\frac{w_{b} c_{b}}{k}} L\right)}+ \\
& e^{-\tilde{\alpha} t / 2} \sum_{n=1}^{\infty}\left(A_{n} \cos \psi_{n} t+B_{n} \sin \psi_{n} t\right) \sin \frac{(n-1 / 2) \pi}{L} x
\end{aligned}
$$

for the hyperbolic equation, while for the parabolic equation one has:

$$
\begin{aligned}
T(x, t)= & \frac{\bar{T}_{s} \cosh \left(\sqrt{\frac{w_{b} c_{b}}{k}}(x-L)\right)}{\cosh \left(\sqrt{\frac{w_{b} c_{b}}{k}} L\right)}+ \\
& \sum_{n=1}^{\infty} A_{n} e^{-\frac{k}{\rho c}\left(\frac{(2 n-1)^{2}}{4 L^{2}} \pi^{2}+\frac{w_{b} c_{b}}{k}\right) t} \sin \frac{(n-1 / 2) \pi}{L} x
\end{aligned}
$$

where

$$
\begin{aligned}
& \tilde{\alpha}=\frac{1}{\tau_{r}}\left(1+\frac{\tau_{r} w_{b} c_{b}}{\rho c}\right) \\
& \psi_{n}=\sqrt{\left(\frac{(n-1 / 2) \pi \sqrt{\frac{k}{\tau_{r} \rho c}}}{L}\right)^{2}-\left(\frac{\rho c-\tau_{r} w_{b} c_{b}}{2 \rho c \tau_{r}}\right)^{2}} \\
& A_{n}=-\frac{(2 n-1) \overline{T_{s}} \pi}{\frac{w_{b} c_{b}}{k} L^{2}+\left(\frac{2 n-1}{2} \pi\right)^{2}} \\
& B_{n}=\frac{\tilde{\alpha}}{2 \psi_{n}} A_{n}
\end{aligned}
$$


Time-history results for the elevated temperature $T_{s}(\mathbf{x}, t)$ at point $A(2.08,1)$ (see Fig. 1(a)) for both $\tau_{r}=0 s$ (Pennes' equation) and $\tau_{r}=20 \mathrm{~s}$ are depicted in Figs. 2-3; the analytical solution is also plotted for comparison. It can be seen that the proposed formulation is capable of controlling the numerical oscillations that appear near discontinuous solutions of the hyperbolic model. Figures 2-3 clearly show the difference between the parabolic $\left(\tau_{r}=0 s\right)$ and hyperbolic $\left(\tau_{r}=20 \mathrm{~s}\right)$ models where, for $\tau_{r}=20 \mathrm{~s}$, the tissue temperature remains zero until the wave front reaches the selected position; afterwards, there is a jump in the solution with the temperature at late times approaching that of the Pennes' result $\left(\tau_{r}=0 s\right)$ where no jump occurs, indicating the same steady-state solution.

Figure 2 clearly shows that results produced by the Stehfest Laplace inversion algorithm are only accurate for $\tau_{r}=0 s$ since the Stehfest algorithm is not capable of accurately representing sharp solutions. Furthermore, Fig. 2 reveals that high numerical oscillations are observed in the HHT- $\alpha$ scheme after the wave front has passed through the observation point, while an accurate solution is obtained by the Crank-Nicolson method. On the other hand, as can be seen in Fig. 3, when $\tau_{r}=20 s$, the ExGA-Stehfest scheme provides unstable results for $\theta=1$ since the stability criterion is not satisfied, while stable results are achieved for $\theta=1.2$ and $\theta=1.4$, indicating that oscillations near the jump are suppressed. To assess the solution close to the jump in a more detailed manner for the results presented in Figs. $2-3$, the temperature results are also presented in Table 1 . It is important to stress that the value of $\theta$ should not be too high since smoothing effects at the discontinuity are clearly observed (notice that when applied to the Pennes' equation, i.e. $\tau_{r}=O s$, the ExGA-Stehfest method also gives accurate solutions, recalling that $\theta=1$ is always adopted in this case). Therefore, the use of the linear $\theta$ method into the standard ExGA formulation has the characteristic of not only decreasing (or even removing) the numerical oscillations, but also stabilizing the solution. A convergence analysis by increasing the number of summation terms $N$ in the Laplace inversion algorithm into the proposed formulation is carried out and the results are shown in Fig. 4. As can be observed results are almost the same according to the graph, implying that the ExGA-Stehfest linear $\theta$ method remains convergent even if the value of $N$ is increased.

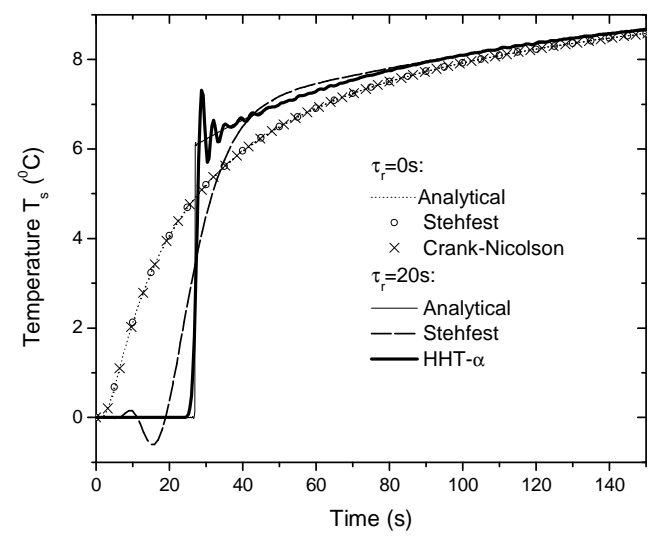

Figure 2. Comparison of the tissue temperature time-history results at point $A(2.08,1)$ for $\tau_{r}=0 \mathrm{~s}$ and $\tau_{r}=20 \mathrm{~s}$ considering the standard CrankNicolson and HHT $-\alpha$ with $\alpha=-1 / 3$ time integration schemes as well as the Stehfest Laplace inversion algorithm.
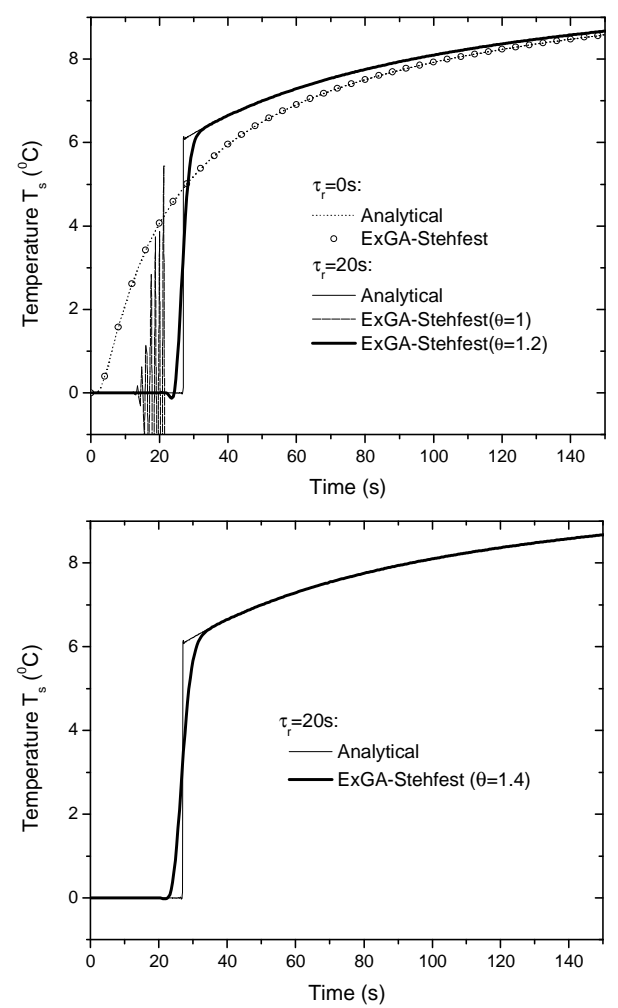

Figure 3. Comparison of the tissue temperature time-history results at point $A(2.08,1)$ for $\tau_{r}=0 s$ and $\tau_{r}=20 s$ considering the ExGA-Stehfest linear $\theta$ method with $\theta=1, \theta=1.2$ and $\theta=1.4$.

Table 1. Temperature results for the hyperbolic model at point $A(2.08,1)$ from $t=20 \mathrm{~s}$ to $t=40 \mathrm{~s}$ considering different numerical schemes.

\begin{tabular}{|c|c|c|c|c|c|}
\hline $\mathrm{t}(\mathrm{s})$ & Analytical $(\mathrm{n}=50000)$ & HHT- $\alpha$ & Stehfest & $\begin{array}{c}\text { ExGA-Stehfest } \\
\theta=1.2\end{array}$ & $\begin{array}{c}\text { ExGA-Stehfest } \\
\theta=1.4\end{array}$ \\
\hline 20 & $5.85071 \times 10^{-6}$ & $5.81534 \times 10^{-10}$ & $\begin{array}{l}3.64975 \\
\times 10^{-1}\end{array}$ & $7.5801 \times 10^{-3}$ & $1.38752 \times 10^{-3}$ \\
\hline 22 & $3.80784 \times 10^{-4}$ & $1.70788 \times 10^{-7}$ & 1.21453 & $-1.82348 \times 10^{-3}$ & $-2.40065 \times 10^{-2}$ \\
\hline 24 & $-5.90609 \times 10^{-4}$ & $4.22401 \times 10^{-4}$ & 2.12777 & $-9.0257 \times 10^{-2}$ & $3.65385 \times 10^{-1}$ \\
\hline 26 & $-1.20596 \times 10^{-4}$ & $4.82645 \times 10^{-1}$ & 3.01028 & 1.84879 & 2.10228 \\
\hline 28 & 6.1249 & 5.72428 & 3.80818 & 4.70248 & 4.32009 \\
\hline 30 & 6.22315 & 6.04386 & 4.49844 & 5.9651 & 5.67037 \\
\hline 32 & 6.31494 & 6.64628 & 5.07783 & 6.27432 & 6.19036 \\
\hline 34 & 6.40324 & 6.29077 & 5.55415 & 6.39532 & 6.37845 \\
\hline 36 & 6.48866 & 6.51374 & 5.9403 & 6.48763 & 6.48324 \\
\hline 38 & 6.5703 & 6.53727 & 6.25061 & 6.57093 & 6.56951 \\
\hline 40 & 6.6488 & 6.67947 & 6.49886 & 6.65201 & 6.64869 \\
\hline
\end{tabular}

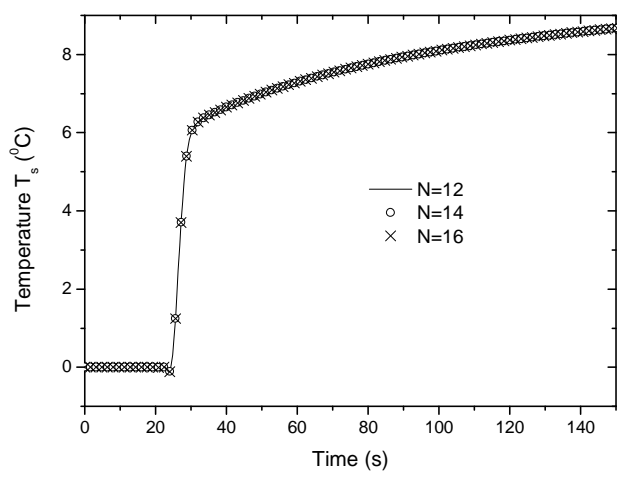

Figure 4. Convergence analysis with respect to the number of summation terms $N$ of the ExGA-Stehfest linear $\theta$ method with $\theta=1.2$ and $\tau_{r}=20 \mathrm{~s}$. 


\section{Multi-layer skin model}

In this second application, a more realistic model with three different layers of tissue (epidermis, dermis and subcutaneous) is considered as depicted in Fig. 5(a). The geometry and the boundary conditions remain the same as those of the previous example, but a more refined mesh (mainly at the epidermis layer) with a total of 4967 four-node quadrilateral elements is employed as shown in Fig. 5(b). The thermal properties of the skin tissue and the thickness of each layer are given in Table 2. Since a finer mesh is employed due to the epidermis layer, the adimensional parameter regarding the time step is increased to $\beta=1.5$, yielding $\Delta t=0.25 \mathrm{~s}$ for $\tau_{r}=20 \mathrm{~s}$.

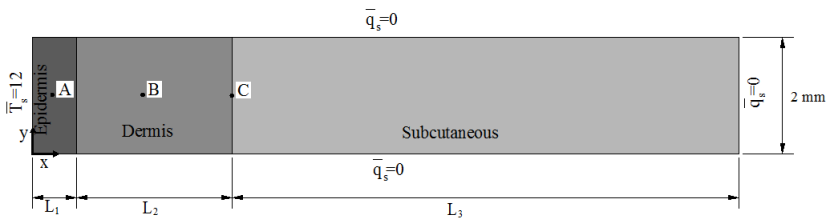

(a)

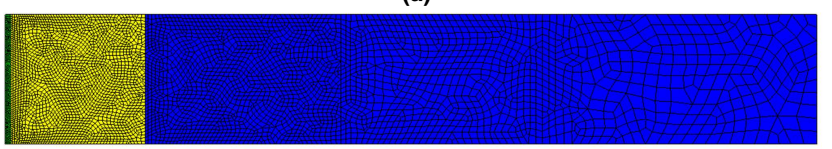

(b)

Figure 5. Heterogeneous biological tissue body: (a) geometry and boundary conditions; (b) domain discretisation.

Table 2. Thickness and thermal properties for the triple layer skin model.

\begin{tabular}{|c|c|c|c|}
\hline Layer & Epidermis & Dermis & Subcutaneous \\
\hline $\mathbf{L}_{\mathrm{i}}(\mathbf{m m})$ & $8.0 \times 10^{-2}$ & 2.0 & 10.0 \\
\hline c $\left(J^{\prime} \cdot g^{-1} \cdot{ }^{0} \mathbf{C}^{-1}\right)$ & 3.6 & 3.4 & 3.06 \\
\hline $\mathrm{k}\left(\mathrm{W} \cdot \mathrm{mm}^{-1} \cdot \mathrm{C}^{-1}\right)$ & $2.6 \times 10^{-4}$ & $5.2 \times 10^{-4}$ & $2.1 \times 10^{-4}$ \\
\hline$\rho\left(\right.$ g.mm $\left.{ }^{-3}\right)$ & $1.2 \times 10^{-3}$ & $1.2 \times 10^{-3}$ & $1.0 \times 10^{-3}$ \\
\hline$c_{b}\left(J \cdot g^{-1} \cdot{ }^{0} C^{-1}\right)$ & 4.2 & 4.2 & 4.2 \\
\hline$w_{b}\left(\mathrm{~g} \cdot \mathrm{mm}^{-3} \cdot \mathrm{s}^{-1}\right)$ & 0.0 & $5.0 \times 10^{-7}$ & $5.0 \times 10^{-7}$ \\
\hline
\end{tabular}

The tissue temperature time history results at point $A(0.04,1)$ (epidermis layer) for $\tau_{r}=0 s$ and $\tau_{r}=20 s$ are plotted in Fig. 6 . Figure 6(a) shows that, because of the jump between the initial condition and the prescribed boundary condition, the CrankNicolson method produces high oscillations in the response, whereas the ExGA-Stehfest scheme for the same time step size gives an accurate solution without any oscillation. The results for $\tau_{r}=20 s$ are plotted in Fig. 6(b). It can be observed again that the ExGA-Stehfest scheme produces no oscillations, while the HHT yields oscillations mainly at the discontinuity. Moreover, since the selected point is very close to the boundary, the wave front rapidly reaches it with the response being quite similar to that of the Pennes equation $\left(\tau_{r}=0 s\right)$ thereafter. Figures $7(\mathrm{a})$ and (b) show the temperature time-history results at points $B(1.08,1)$ (dermis layer) and $C(2.08,1)$ (interface between dermis and subcutaneous layers), respectively. For comparison purposes, results for $\tau_{r}=10$ $s$ are also plotted in Fig. 7 to show the influence of this parameter in the solution. First, it can be seen that the non-homogeneous medium significantly affects the response, where a more complex response is obtained when compared to the homogeneous case of the previous example. This is due to superposition effects originated from the reflected thermal waves at the interface between layers (observe that, as in the first example, accurate results are obtained by the ExGA-Stehfest method for $\tau_{r}=0 \mathrm{~s}$ ). Finally, in the case of $\tau_{r}=10 s$ the wave front reaches points B and $\mathrm{C}$ faster than that of $\tau_{r}=20 \mathrm{~s}$. This is expected since decreasing the value of $\tau_{r}$ implies a rise in the thermal wave speed according to its expression. Table 3 presents the temperature results from $t=20 \mathrm{~s}$ to $t=40 \mathrm{~s}$ in order to compare the numerical results more clearly for the two values of $\tau_{r}$. The temperature distribution at time instant $t=15.25 \mathrm{~s}$, for the parabolic and hyperbolic models, is displayed in Fig. 8. It is clearly observed that the temperature distribution for the parabolic model (infinite wave speed) reaches the deeper tissue faster than that of the hyperbolic one (finite wave speed).

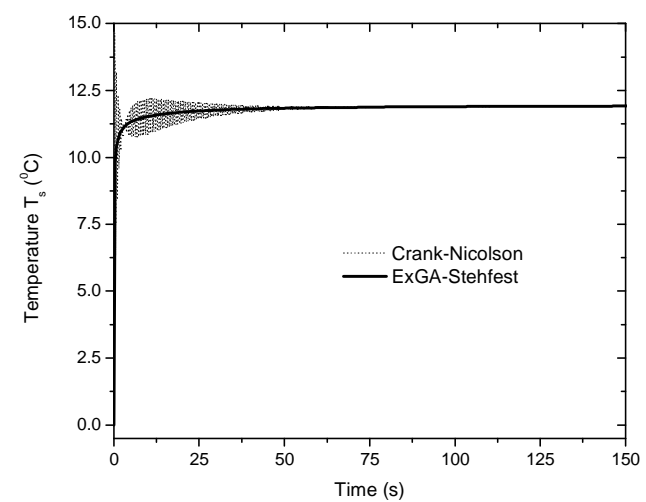

(a)

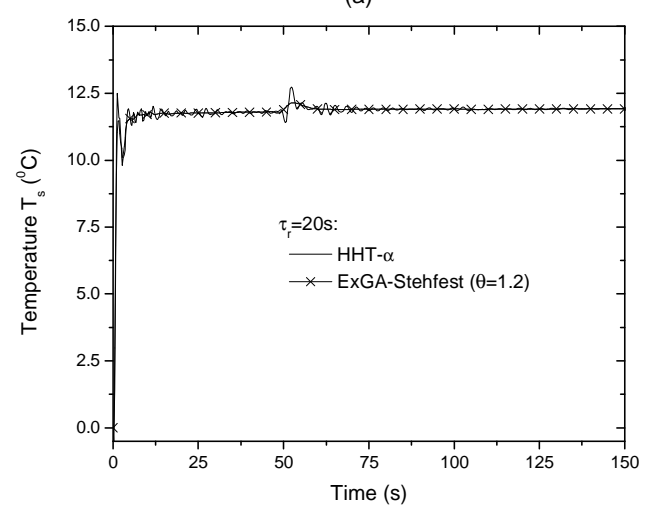

(b)

Figure 6. Comparison of the tissue temperature time-history results at point $A(0.04,1)$ for $\tau_{r}=0 s$ and $\tau_{r}=20 \mathrm{~s}$ : (a) Crank-Nicolson and EXGAStehfest schemes; (b) HHT $-\alpha$ with $\alpha=-1 / 3$ and ExGA-Stehfest with $\theta=$ 1.2 schemes.

Table 3. Temperature results for the hyperbolic model at point $B(1.08,1)$ from $\mathrm{t}=\mathbf{2 0} \mathrm{s}$ to $\mathrm{t}=\mathbf{4 0} \mathrm{s}$.

\begin{tabular}{|l|c|c|c|c|}
\hline $\mathrm{t}(\mathrm{s})$ & $\begin{array}{c}\text { HHT- } \alpha \\
\tau_{r}=10 s\end{array}$ & $\begin{array}{c}\text { ExGA-Stehfest } \\
\tau_{r}=10 s\end{array}$ & $\begin{array}{c}\text { HHT- } \alpha \\
\tau_{r}=20 s\end{array}$ & $\begin{array}{c}\text { ExGA-Stehfest } \\
\tau_{r}=20 s\end{array}$ \\
\hline 20 & 7.86997 & 7.88996 & 8.3172 & 8.41075 \\
\hline 22 & 8.01969 & 8.01031 & 8.54977 & 8.55601 \\
\hline 24 & 8.12319 & 8.1191 & 8.58252 & 8.61742 \\
\hline 26 & 8.22444 & 8.26474 & 8.69824 & 8.69083 \\
\hline 28 & 8.82423 & 8.82098 & 8.67786 & 8.74571 \\
\hline 30 & 9.13848 & 9.23164 & 8.75932 & 8.79887 \\
\hline 32 & 9.38728 & 9.43417 & 8.85777 & 8.85546 \\
\hline 34 & 9.58861 & 9.57363 & 8.89253 & 8.91656 \\
\hline 36 & 9.73481 & 9.69652 & 8.96901 & 8.89962 \\
\hline 38 & 9.83824 & 9.80875 & 8.76211 & 9.29635 \\
\hline 40 & 9.94776 & 9.91499 & 10.0494 & 9.90154 \\
\hline
\end{tabular}




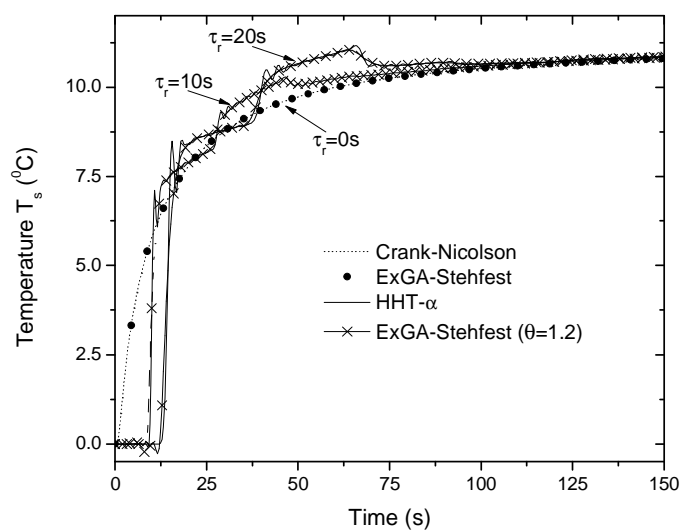

(a)

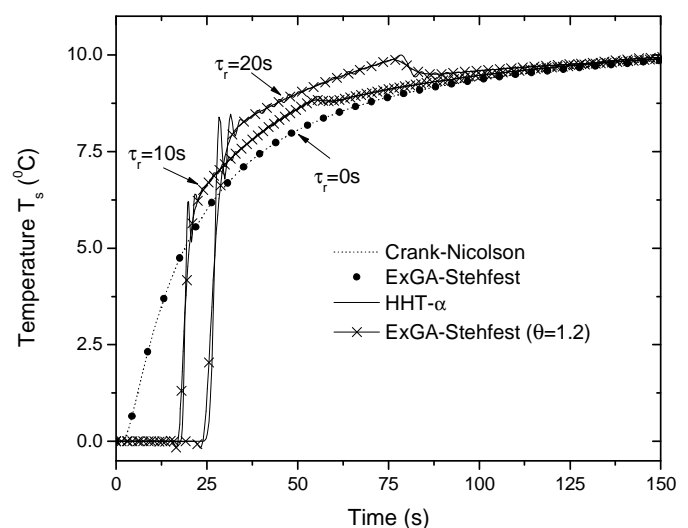

(b)

Figure 7. Comparison of the tissue temperature time-history for $\tau_{r}=0 \mathrm{~s}$ $\tau_{r}=10 s$ and $\tau_{r}=20 s$ considering the Crank-Nicolson, HHT- $\alpha$ with $\alpha=$ $-1 / 3$ and ExGA-Stehfest linear $\theta$ with $\theta=1.2$ schemes: (a) point $B(1.08,1)$; (b) point $C(2.08,1)$.

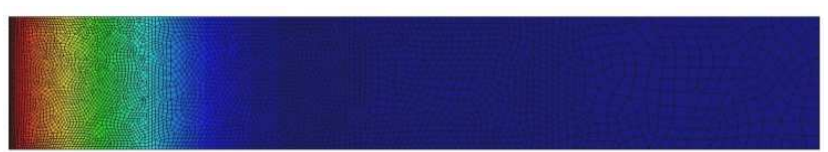

(a)

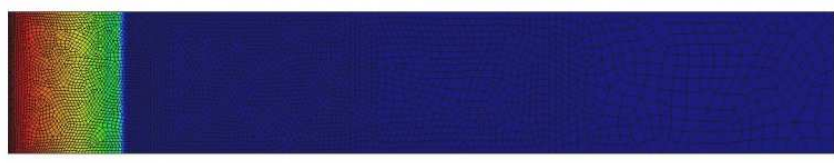

(b)

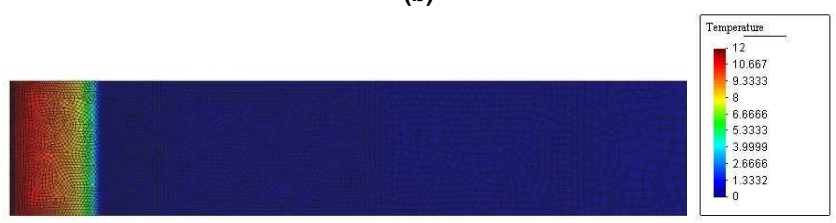

(c)

Figure 8. Tissue temperature field at time instant $t=15.25 \mathrm{~s}$ : (a) $\tau_{r}=0 \mathrm{~s}$ considering the ExGA-Stehfest scheme; (b) $\tau_{r}=10 \mathrm{~s}$ considering the ExGA-Stehfest linear $\theta$ scheme with $\theta=1.2$; (c) $\tau_{r}=20 \mathrm{~s}$ considering the ExGA-Stehfest linear $\theta$ scheme with $\theta=1.2$.

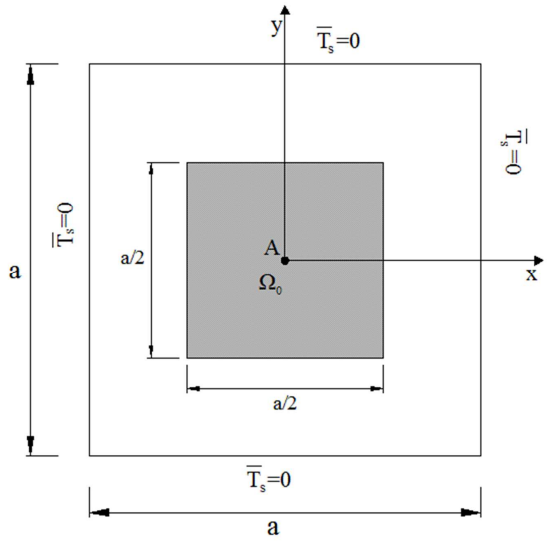

Figure 9. Geometry and boundary conditions of the square biological tissue body under initial temperature field over the domain $\Omega_{0}$.

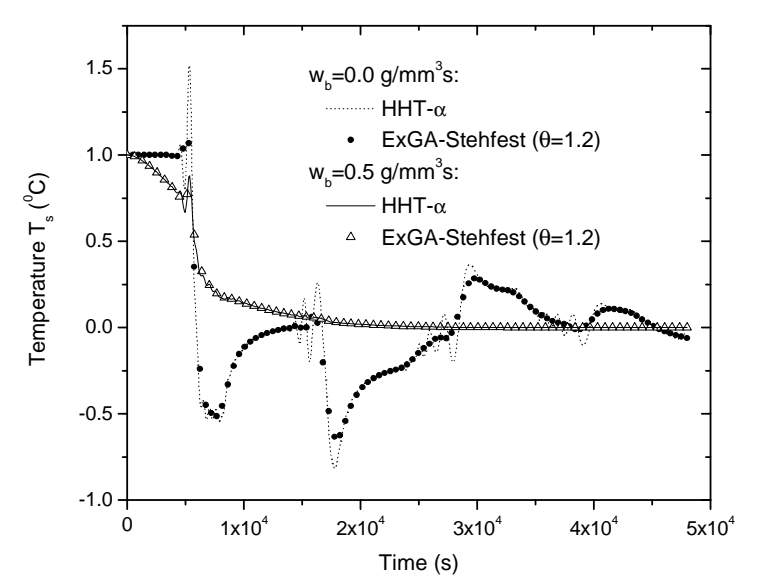

(a)

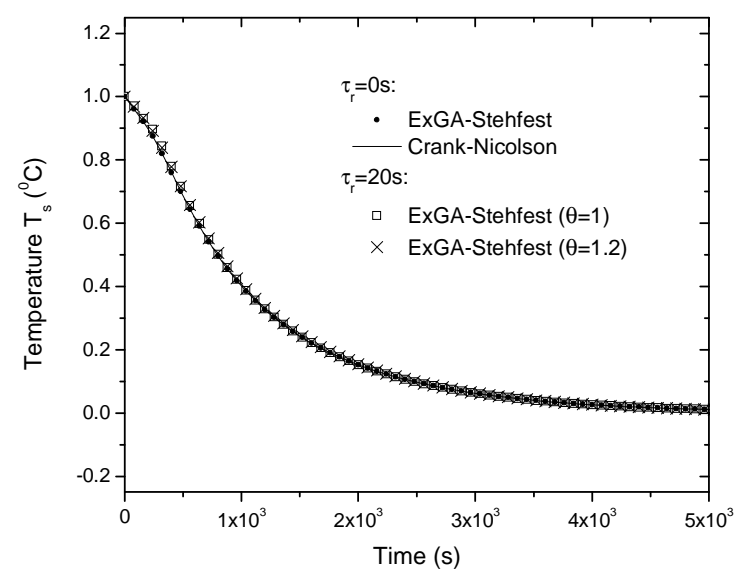

(b)

Figure 10. Comparison of the tissue temperature time-history results at point $A(0,0)$ using a time step size $\Delta t=80 \mathrm{~s}$ : (a) HHT- $\alpha$ with $\alpha$ $=-1 / 3$ and ExGA-Stehfest with $\theta=1.2$ schemes considering $\tau_{r}=10^{4} \mathrm{~s}$, $w_{b}=0.0 \mathrm{~g} \cdot \mathrm{mm}^{-3} \cdot \mathrm{s}^{-1}$ and $w_{b}=5.0 \times 10^{-7} \mathrm{~g} \cdot \mathrm{mm}^{-3} \cdot \mathrm{s}^{-1}$; (b) Crank-Nicolson and ExGA-Stehfest with $\theta=1$ and $\theta=1.2$ schemes considering $\tau_{r}=0 \mathrm{~s}$, $\tau_{r}=20 s$ and $w_{b}=5.0 \times 10^{-7} \mathrm{~g} \cdot \mathrm{mm}^{-3} \cdot \mathrm{s}^{-1}$. 


\section{Two-dimensional bioheat model}

This example is concerned with the thermal analysis in a square biological tissue with dimension $a=80 \mathrm{~mm}$ subjected to an initial temperature condition $T_{s 0}(\mathbf{x})=1.0^{\circ} \mathrm{C}$ prescribed over the domain $\Omega_{0}=\{(x, y):-a / 2 \leq x \leq a / 2,-a / 2 \leq y \leq a / 2\}$ as sketched in Fig. 9. Due to the symmetry of the problem, only one quarter of the domain needs to be modeled, where a uniform mesh of 2500 fournode quadrilateral elements is employed to spatially discretize the model. The thermal properties of the tissue have been chosen as $\rho=1.0 \times 10^{-3} \mathrm{~g} / \mathrm{mm}^{3}, \quad c=c_{b}=4.2 \mathrm{~J} / \mathrm{g}^{0} \mathrm{C}, \quad k=5.0 \times 10^{-4}$ $W / \mathrm{mm}^{\circ} \mathrm{C}$ and $w_{b}=5.0 \times 10^{-7} \mathrm{~g} / \mathrm{mm}^{3} \mathrm{~s}$. The numerical analysis is performed taking into consideration different values for thermal relaxation time and blood perfusion rate and the time step is selected such that $\beta=0.345$.

First the thermal relaxation time and the blood perfusion rate are taken, respectively, as $\tau_{r}=10^{4} s$ and $w_{b}=0.0 \mathrm{~g} / \mathrm{mm}^{3} \mathrm{~s}$, which is common in cryogenic surgery; see for instance ( $\mathrm{Lu}$ et al., 1998) the time-history results at point $A(0,0)$ (see Fig. 9) that are plotted in Fig. 10(a) (results for the same thermal relaxation time but with a blood perfusion rate $w_{b}=5.0 \times 10^{-7} \mathrm{~g} / \mathrm{mm}^{3} \mathrm{~s}$ are also depicted in Fig. 10(a) for comparison). As can be observed, the blood perfusion rate $w_{b}=5.0 \times 10^{-7} \mathrm{~g} / \mathrm{mm}^{3} \mathrm{~s}$ produces a more damping solution than the null perfusion rate (notice that the ExGA-Stehfest method with $\theta=1.2$ for $w_{b}=0.0 \mathrm{~g} / \mathrm{mm}^{3} \mathrm{~s}$ provides a more clear solution without the high oscillations presented in the HHT scheme results). Next when the thermal relaxation time is assumed to be $\tau_{r}=20 \mathrm{~s}$, the results become very similar to that of the Pennes' equation $\left(\tau_{r}=0 \mathrm{~s}\right)$ as shown in Fig. 10(b). It is worth pointing out that since the wave phenomenon does not appear when $\tau_{r}=20 \mathrm{~s}$, the ExGA-Stehfest method with $\theta=1$ becomes stable.

\section{Conclusions}

In this paper, a hybrid time-Laplace domain technique based on numerical Green's functions has been proposed for the solution of hyperbolic bioheat transfer models. As an additional advantage of the proposed formulation, the parabolic model can also be solved by just setting $\tau_{r}=0$ without considering any further numerical considerations. The novelty of the present work is the introduction of the linear $\theta$ method into the standard ExGA time-marching scheme, establishing the so-called ExGA-linear $\theta$ method. In the ExGA-linear $\theta$ method, the temperature response is firstly computed at time $t_{k+\theta}=(k+\theta) \Delta t$ from the previous known solution at time $t_{k}=k \Delta t$, and then linearly interpolated to obtain the solution at time $t_{k+1}=(k+1) \Delta t$. As a result, only the Green's functions represented by the Green's matrices at time $t_{\theta}=\theta \Delta t$ need to be computed; herein, this is accomplished by the Stehfest Laplace inversion algorithm.

On the basis of the numerical examples, it can be stated that accurate results are produced by the ExGA-Stehfest linear $\theta$ method. Furthermore, the proposed formulation with $\theta>1$ can be successfully employed not only to deal with sharp fronts, but also to stabilize the numerical solution in the hyperbolic model. Although numerical oscillations near discontinuities can be suppressed when $\theta>1$ is adopted, the value of $\theta$ should not be too high to avoid the introduction of smoothing effects. For the parabolic model, however, the parameter $\theta=1$ may be adopted as smooth solutions are always expected. Thus, an effective unified numerical technique to solve both parabolic and hyperbolic bioheat problems can be developed through the proposed formulation.

\section{Acknowledgements}

The first author gratefully acknowledges the support of the Brazilian agency CAPES (Coordenação de Aperfeiçoamento de Pessoal de Nivel Superior) in funding this work at Brunel University through the scholarship No. BEX4623/08-8.

\section{References}

Beck, J.V., Cole, K.D., Haji-Sheikh, A. and Litkouhi B., 1992, "Heat conduction using Green's functions", Hemisphere, Washington, DC.

Cattaneo, C., 1958, "A Form of Heat Conduction Equation which Eliminates the Paradox of Instantaneous Propagation”, Compte Rendus, Vol. 247, pp. 431-433.

Chan, C.L., 1992, "Boundary Element Method Analysis for the Bioheat Transfer Equation", ASME J. Biomech. Eng., Vol. 114, pp. 358-65.

Dai, W., Yu, H. and Nassar, R., 2004, "A Fourth-order Compact Finite Difference Scheme for Solving a 1-D Pennes Bioheat Transfer Equation in a Triple-layered Skin Structure", Numer. Heat Tran. Part B, Vol. 46, pp. 447-461.

Deng, Z.S. and Liu, J., 2002, "Analytical Study on Bioheat Transfer Problems with Spatial or Transient Heating on Skin Surface or Inside Biological Bodies", ASME J. Biomech Eng., Vol. 124, pp. 638-649.

Gao, B., Langer, S. and Corry, P.M., 1995, “Application of the TimeDependent Green's Function and Fourier Transforms to the Solution of the Bioheat Equation", Int. J. Hyperth., Vol. 11, pp. 267-285.

Hughes, T.J.R., 1987, "The Finite Element Method: Linear Static and Dynamic Finite Element Analysis", Prentice-Hall, Englewood Cliffs, New Jersey.

Khanafer, K. and Vafai, K., 2009, "Synthesis of Mathematical Models Representing Bioheat Transport”, in: Minkowycz W.J., Sparrow E.M. (eds.), Advances in Numerical Heat Transfer, Vol. 3, Taylor \& Francis, New York, pp. $1-28$.

Li, F.F., Liu, J. and Yue, K., 2009, "Exact Analytical Solution to ThreeDimensional Phase Change Heat Transfer Problems in Biological Tissues Subject to Freezing", App. Math. Mech. (English Edition), Vol. 30, pp. 63-72.

Liu, J., Chen, X. and Xu, L.X., 1999, "New Thermal Wave Aspects on Burn Evaluation of Skin Subjected to Instantaneous Heating", IEEE Trans. Biomed. Engrg., Vol. 46, pp. 420-428.

Liu, J. and Xu, L.X., 2000, "Boundary Information based Diagnostics on the Thermal States of Biological Bodies", Int. J. Heat Mass Tran., Vol. 43, pp. 2827-2839.

Liu, K.C., 2008, "Thermal Propagation Analysis for Living Tissue with Surface Heating”, Int. J. Thermal Sci., Vol. 47, pp. 507-513.

Loureiro, F.S., 2007, "Time integration methods based on the calculation of numerical Green's functions by the finite element method" (in Portuguese). Master's Thesis, UFRJ-COPPE, Rio de Janeiro, Brazil.

Loureiro, F.S., Mansur, W.J. and Vasconcellos, C.A.B., 2009, “A Hybrid Time/Laplace Integration Method Based on Numerical Green's Functions in Conduction Heat Transfer", Comput. Meth. Appl. Mech. Eng., Vol. 198, pp. 2662-2672.

Loureiro, F.S. and Mansur, W.J., 2009a, “An Efficient Hybrid TimeLaplace Domain Method for Elastodynamic Analysis Based on the Explicit Green's Approach”, Int. J. Solids and Struct., Vol. 46, pp. 3093-3102.

Loureiro, F.S. and Mansur W.J., 2009b, "A Novel Higher-Order Time Integration Method Using the Modified Explicit Green's Approach for Linear Parabolic Problems", Num. Heat Tran., Part B, Vol. 56, pp. 211-230.

Loureiro, F.S. and Mansur, W.J., 2010, "A Novel Time-Marching Scheme Using Numerical Green's Functions: A Comparative Study for the Scalar Wave Equation", Comput. Meth. Appl. Mech. Eng., Vol. 199, pp. 1502-1512.

Loureiro, F.S., Oyarzúna, P., Santos, J.P.L., Mansur, W.J. and Vasconcellos, C.A.B., 2010, “A Hybrid Time/Laplace Domain Method Based on Numerical Green's Functions Applied to Parabolic and Hyperbolic Bioheat Transfer Problems", CILAMCE 2010/Mecánica Computacional, Vol. XXIX, pp. 5599-5611. 
Lu, W.Q., Liu, J. and Zeng Y., 1998, "Simulation of The Thermal Wave Propagation in Biological Tissues By the Dual Reciprocity Boundary Element Method", Eng. Anal. Bound. Elem., Vol. 22, pp. 167-174.

Mansur, W.J., 1983, "A Time-Stepping Technique to Solve Wave Propagation Problems Using the Boundary Element Method", PhD thesis, University of Southampton, England.

Mansur, W.J., Loureiro, F.S., Soares, D. Jr. and Dors, C., 2007, "Explicit Time-Domain Approaches Based on Numerical Green's Functions Computed By Finite Differences - The ExGA Family", J. Comp. Phys., Vol. 227, pp. 851-870.

Newman, W.H., Lele, P.P. and Bowman, H.F., 1990, "Limitations and Significance of Thermal Washout Data Obtained During Microwave and Ultrasound Hyperthermia", Int. J. Hyperth., Vol. 6, pp. 771-784

Özen, S., Helhel, S. and Cerezci O., 2008, "Heat Analysis of Biological Tissue Exposed to Microwave by Using Thermal Wave Model of Bio-heat Transfer (TWMBT)", Burns, Vol. 34, pp. 45-49.

Presgrave, A.V., Guedes, R.O.C. and Scofano Neto, F., 2009, "Integral Transform Solution to the Endometrial Ablation Problem", J. Braz. Soc. Mech. Sci. \& Eng., Vol. XXXI, No. 2, pp. 117-124. ISSN 1678-5878.

Rubinsky, B., 2006, "Numerical Bioheat Transfer", in: Minkowycz W.J., Sparrow E.M., Murthy J.Y. (eds.), Handbook of Numerical Heat Transfer, 2nd ed., John Wiley and Sons, New Jersey, pp. 851-893.
Stehfest, H., 1970, "Algorithm 368: Numerical Inversion of Laplace Transform”, Commun. Assoc. Comput. Mech., Vol. 13, pp. 47-49.

Torvi, D.A. and Dale, J.D., 1994, "A Finite Element Model of Skin Subjected to a Flash Fire”, ASME J. Biomech. Eng., Vol. 116, pp. 250-255.

Tunga, M.M., Trujillo, M., López Molina, J.A., Rivera, M.J. and Berjano, E.J., 2009, "Heat Analysis of Biological Tissue Exposed to Microwave by Using Thermal Wave Model of Bio-heat Transfer", Math. Comp. Model, Vol.. 50, pp. 665-672.

Wrobel, L.C., 2002, "The Boundary Element Method: Applications in Thermo-Fluids and Acoustics", John Wiley and Sons, England.

Wu, J.P. and Chu, H.S., 1999, "Propagation and Reflection of Thermal Waves in a Rectangular Plate", Numer. Heat Tran., Part A, Vol. 36, pp. 51-74

Xu, F., Seffen, K.A. and Lu T.J., 2008, "Non-Fourier Analysis of Skin Biothermomechanics”, Int. J. Heat Mass Tran., Vol. 51, pp. 2237-2259.

Yu, G., Mansur, W.J., Carrer, J.A.M. and Gong, L., 1998, "A Linear $\theta$ Method Applied to 2D Time-domain BEM", Comm. Num. Meth. Eng., Vol. 14, pp. 1171-1179.

Zhou, J., Zhang, Y. and Chen J.K., 2009, "An Axisymmetric DualPhase-Lag Bioheat Transfer Model for Laser Heating of Living Tissues", Int. J. Therm. Sci.,Vol. 48, pp. 1477-1485. 